\title{
Conocimientos, actitudes y prácticas sobre la enfermedad de Chagas en Aguazul Casanare
}

\author{
Knowledge, attitudes, and practices about Chagas disease in Aguazul, Casanare (Colombia)
}

\author{
Leidy Constanza Cano-Rodríguez' orcid.org/0000-0003-0686-0303 \\ Julie Liliana Orjuela-Vargas ${ }^{2,3}$ orcid.org/0000-0001-6952-226X \\ Ángela Liliana Monroy-Díaz 2,3* orcid.org/0000-0002-3420-9123
}

\begin{abstract}
1. Programa de Bacteriología y Laboratorio Clínico, Universidad de Boyacá. Tunja, Colombia.
2. Facultad de Ciencias de la Salud, Universidad de Boyacá. Tunja, Colombia.

3. Grupo de Investigación del Programa de Bacteriología y Laboratorio Clínico (GRIBAC), Universidad de Boyacá. Tunja, Colombia.
\end{abstract}

\section{Resumen}

Introducción: En Colombia la enfermedad de Chagas ocasionada por el protozoo Trypanosoma cruzi, es considerada un problema de salud pública, que requiere una línea base para que las comunidades puedan enfrentar esta patología. Objetivo: Determinar los conocimientos, actitudes y prácticas de los habitantes del municipio de Aguazul-Casanare frente a la enfermedad de Chagas. Materiales y métodos: Se realizó un estudio descriptivo de corte transversal, se encuestaron a 389 familias del municipio; se indagó sobre factores sociodemográficos, epidemiológicos, conocimientos, actitudes y prácticas. Resultados: Participaron 221 mujeres $(56,8 \%)$ y 168 hombres $(43,2 \%)$. El 31,4\% de los participantes tienen conocimientos óptimos, 31,6\% buenos. Se observó actitudes óptimas en el 85,9\%; el 56,3\% tiene malas prácticas y el 43,3\% entre prácticas buenas y regulares. Conclusiones: Se determinó que a pesar de que la comunidad tiene buenas actitudes frente a la prevención de la infección, existe un bajo porcentaje en conocimiento óptimo, que se refleja en malas prácticas. Por ello, resulta importante establecer acciones de educación encaminadas al fortalecimiento de conocimientos relacionados con la patología que impacte en la reducción de la endemia.

Palabras clave: Enfermedad de Chagas; conocimientos; actitudes y práctica en salud; Trypanosoma cruzi; transmisión. (Fuente: DeCS, Bireme).

\begin{abstract}
Introduction: Chagas disease is caused by the protozoan Trypanosoma cruzi. It is considered a public health problem in Colombia that requires a baseline to face this pathology. Objective: To identify knowledge, attitudes and practices of the inhabitants of the municipality of Aguazul-Casanare to handle Chagas disease. Materials and methods: A descriptive cross-sectional study was conducted on 389 families of the municipality. Sociodemographic and epidemiologic factors as well as knowledge, attitudes and practices were investigated. Results: 221 women (56.8\%) and 168 men (43.2) participated in the study. 31.4\% and 31.6\% of participants have optimal and appropriate knowledge, respectively. Optimal attitudes were observed in $85.9 \%$ of people, whereas $56.3 \%$ show improper practices, and $43.3 \%$ have good and regular practices. Conclusions: Even though, the community has positive attitudes towards prevention of the infection, there is a reduced percentage of people showing optimal knowledge, which is reflected in unhealthy practices. Thus, it is important to establish educational actions aimed at strengthening knowledge related to the Chagas that have an impact on the reduction of this endemic disease.
\end{abstract}

Keywords: Chagas disease; health knowledge; attitudes; practice; Trypanosoma cruzi; transmission. (Source: DeCS, Bireme).

\footnotetext{
*Autor de correspondencia

Angela Liliana Monroy-Díaz

e-mail: almonroy@uniboyaca.edu.co
} 


\section{Introducción}

La enfermedad de Chagas es causada por el parásito Trypanosoma cruzi (T. cruzi), con diversas formas de trasmisión, una de ellas es la ocasionada por insectos Triatomíneos. Es considerada una enfermedad desatendida en América Latina y un reciente problema de salud pública en países no endémicos a causa de otras formas de trasmisión como la vertical y transfusional(1). Esta patología está presente en 21 países de Latinoamérica(2) y se estima que en el mundo existen entre 6 a 7 millones de personas infectadas con este parásito(3); así mismo se estima que aproximadamente 100 millones de personas viven en áreas de riesgo de la infección ${ }^{(4)}$ y es responsable de alrededor de 12 mil muertes al año.

A pesar de las acciones que se establecen con el objetivo de interrumpir la trasmisión(5), en Colombia uno de los departamentos de mayor endemia es Casanare con prevalencias que oscilan entre 16,9\% y el $30 \%{ }^{(6)}$ además de los brotes por trasmisión oral que son causa importante de mortalidad(7).

Dentro de los factores de riesgo que favorecen el proceso de infección, está la presencia de espacios peri-domiciliarios en los cuales habitan animales que sirven como reservorio del parásito ${ }^{(8)}$; los factores sociodemográficos como la marginación, la pobreza, vivienda deficiente, escasos o nulos servicios de saneamiento básico y la inoportunidad de acceso a servicios de salud por distintas variables ${ }^{(9)}$. Se ha descrito que el conocimiento de la población respecto a la enfermedad podría ser indispensable para prevenirla o determinar la infección en fase aguda e impactar en la reducción de la endemia ${ }^{(6-9)}$.

Una herramienta potencial de salud pública para la intervención efectiva de las comunidades podrá establecerse a través de la participación de la población y los estudios de conocimientos, actitudes y prácticas (CAPS), que permiten abordar problemáticas sociales, a través del diagnóstico de temas de interés para las poblaciones objeto de estudio, ofreciendo un avance y apoyo para la promoción de la salud(10). Estos diagnósticos de percepción frente a las enfermedades en las comunidades generan líneas de base para determinar políticas o estrategias de control.

El municipio de Aguazul, ubicado en el Departamento de Casanare, se caracteriza por presentar una temperatura aproximada de $26^{\circ} \mathrm{C}$ y se encuentra a una altitud de 2.763 m.s.n.m., factores que favorecen el ciclo de reproducción vectorial(11), por tal razón el objetivo del presente estudio fue determinar los conocimientos, actitudes y prácticas de los habitantes del municipio de Aguazul-Casanare frente a la enfermedad de Chagas.

\section{Materiales y métodos}

\section{Diseño de estudio}

Se llevó a cabo un estudio descriptivo de corte transversal, durante el año 2019.

\section{Población y muestra}

Para la selección de la muestra, fue necesario tomar la población total mayor de dieciocho años de edad del municipio de Aguazul, correspondiente a 25.880 personas (Fuente SISBEN Aguazul), para calcular la muestra se utilizó un referente de frecuencia del $37 \%{ }^{(12)}$ y por medio del programa OpenEpi, se determinó un tamaño de muestra total de 389 domicilios, con un porcentaje de pérdida del $10 \%$ y con un intervalo de confianza del 95\%. Posteriormente, se generó una afijación proporcional para establecer una muestra equiparable entre las familias de zona urbana y rural.

Como criterios de selección se tuvo en cuenta una persona mayor de edad por vivienda y se excluyeron aquellas personas que poseían algún limitante cognitivo que le pudiera impedir el diligenciamiento del instrumento.

\section{Técnicas e instrumentos de recolección de información}

El instrumento para la recolección de datos, cuenta con 27 preguntas divididas en cuatro dimensiones: la primera enfocada a la socioepidemiología, la demás indagan acerca de los conocimientos, las actitudes y las prácticas de los habitantes del municipio de Aguazul en relación a la enfermedad de Chagas. Para las preguntas que involucraban el vector, se contó con un stock de insectos, facilitando el reconocimiento del mismo. El instrumento usado tuvo validación de contenido y constructo enfocado a poblaciones donde la enfermedad es endémica(13), con un índice Kappa entre 0,7-0,8 y un alfa de Cronbach de 0,85. Para determinar los CAPS se asignaron valores numéricos (Tabla 1). 
Tabla 1. Escala de medición y puntuación para evaluar CAPS

\begin{tabular}{llr}
\hline Referente & \multicolumn{1}{c}{ Escala } & Puntuación \\
\hline Conocimientos & Óptimo & $8-10$ \\
& Bueno & $6-7$ \\
& Regular & $3-5$ \\
& Malo & $0-2$ \\
Actitudes & Óptimo & $17-21$ \\
& Bueno & $15-16$ \\
& Regular & $12-15$ \\
Prácticas & Malo & $5-11$ \\
& Óptimo & 10 \\
& Bueno & $8-9$ \\
& Regular & $6-7$ \\
& Malo & $0-5$ \\
\hline
\end{tabular}

\section{Procesamiento y análisis de la información}

Para la operacionalización de la información recolectada se generó una matriz en una hoja de cálculo y se procesó en el programa estadístico SPSS ${ }^{\circledR}$ 22 , para el análisis de cada una de las variables se utilizó la estadística descriptiva.

\section{Consideraciones éticas}

La presente investigación fue aprobada por el Comité de Ética y Bioética de la Universidad de Boyacá mediante el memorando 00142019 y fue catalogada como una investigación sin riesgo según la Resolución 008430 de 1993, dado que no se realizó ningún tipo de modificación en la variables biológicas, fisiológicas, sicológicas o sociales de las personas que decidieron participar en la investigación. Previo a la recolección de la información, los participantes diligenciaron el consentimiento informado, el cual le permitió a la persona involucrada, conocer cuáles serían los posibles riesgos y beneficios a los cuales estaría expuesto durante el desarrollo del estudio.

\section{Resultados}

Se entrevistó a 389 representantes de domicilios, de ellos, 221 personas (56,8\%) eran mujeres y 168 $(43,2 \%)$ hombres, la población presentó una edad mínima de 18 años y máxima de 77 , con una media de 36,2 años (desviación estándar: $\pm 12,7$ ), el 28,8\% pertenecía al área rural del municipio, respecto al nivel de escolaridad la mayoría tenía estudios en secundaria con un $44,2 \%$ seguido de primaria con $25,2 \%$ y pregrado universitario en un $12,3 \%$.

Se identificó que el 71,5\% de la población reconoció el insecto trasmisor del parásito T. cruzi, y en su mayoría lo conocían con el nombre común de "pito", el 30,8\% de los encuestados mencionó haber visto el insecto cerca a su domicilio, y 29 (7,5\%) personas refieren haber sido picados por este insecto. Por otra parte, se determinó que el $8,5 \%$ de la población involucrada en la investigación ha recibido transfusiones sanguíneas importantes y a pesar de que este es uno de los mecanismos de trasmisión de la infección para el presente estudio no se evidenciaron casos de infección por este medio.

El 23,1\% de los habitantes menciona que en algún momento han tenido familiares diagnosticados con la enfermedad de Chagas, principalmente abuelos o padres y el $69,7 \%$ de las personas cuentan con algún animal ya sea en su domicilio o peri-domicilio. Frente a los distintos tipos de material empleados para la construcción de las viviendas, se encontró que el $55,8 \%$ de las casas tienen piso en cemento, el 75,8\% tienen techo con tejas de fibrocemento y el $65,8 \%$ de las paredes son en ladrillo.

Respecto al conocimiento frente a la enfermedad de Chagas, se determinó que el $60,7 \%$ de las personas conoce o ha oído hablar de esta enfermedad. De las personas que menciona conocerla el $67,6 \%$ refieren que la forma de transmisión se da por vía vectorial: el $72,2 \%$ explica que los sitios donde se encuentra el insecto trasmisor es en partes boscosas o casas de palma, el $22 \%$ explica que son los dormitorios, sin embargo, el $50,4 \%$ no tienen conocimiento de la fuente de alimento del vector. El 68,4\% sabe que esta infección afecta el cuerpo destacando el corazón como órgano blanco y el $26,7 \%$ afirma que esta enfermedad podría ser tratada y tiene cura.

En relación a las actitudes de la comunidad, el 78,7\% dice que participaría activamente en la búsqueda del vector en sus alrededores, el 83,8\% precisó que aceptaría cursos o charlas de la enfermedad de Chagas. Al indagar qué haría ante la picadura del insecto, un $82,2 \%$ acudiría de inmediato al centro médico y sólo un pequeño porcentaje menciona la utilización de remedios caseros, además el 91,8\% aceptaría un examen de sangre con la intención de conocer si son portadores del parásito T. cruzi.

Finalmente, como parte de las prácticas se observó que el 74,8\% nunca ha escuchado charlas acerca de la enfermedad de Chagas, el 52,4\% menciona que no utiliza toldillo al momento de dormir, el $64,5 \%$ usa insecticidas con el fin de contrarrestar cualquier insecto dentro de su domicilio, y el $98,7 \%$ en caso de ser diagnosticados con la afección aceptarían someterse a algún tipo de tratamiento (Tabla 2). 
En relación a los CAPS en esta comunidad se evidenció que la población presenta conocimientos óptimos en un $31,4 \%$, actitudes óptimas en un $85,9 \%$ y malas prácticas en un 56,3 \% (Figura 1).

Tabla 2. CAPS frente a la enfermedad de Chagas en Aguazul Casanare

\begin{tabular}{|c|c|c|c|c|c|}
\hline \multicolumn{3}{|r|}{ Variable } & \multirow{2}{*}{$\begin{array}{l}\begin{array}{l}\text { Mujeres } \\
\text { n (\%) }\end{array} \\
152(68,8)\end{array}$} & \multirow{2}{*}{$\begin{array}{c}\begin{array}{c}\text { Hombres } \\
\text { n (\%) }\end{array} \\
126(75)\end{array}$} & \multirow{2}{*}{$\begin{array}{l}\text { Total } \\
\text { n (\%) } \\
278(71,5)\end{array}$} \\
\hline Factores socio- & & & & \\
\hline epidemiológicos & \multicolumn{2}{|c|}{ Conoce el insecto como "pito" } & $147(66,5)$ & $122(72,6)$ & $269(69,2)$ \\
\hline & \multicolumn{2}{|c|}{ Ha visto el insecto en su domicilio o alrededores } & $65(29,4)$ & $55(32,7)$ & $119(30,8)$ \\
\hline & \multicolumn{2}{|c|}{ Ha sido picado por el insecto } & $20(9,0)$ & $9(5,3)$ & $275(7,9)$ \\
\hline & \multicolumn{2}{|c|}{ Ha recibido trasfusiones de sangre o trasplantes } & $20(9,1)$ & $13(7,8)$ & $33(8,5)$ \\
\hline & \multicolumn{2}{|c|}{ Tiene familiares con diagnóstico de Chagas } & $59(26,7)$ & $31(18,4)$ & $90(23,1)$ \\
\hline & \multicolumn{2}{|c|}{ Tiene animales en su domicilio } & $160(72,4)$ & $111(66,1)$ & $271(69,7)$ \\
\hline & \multirow{4}{*}{$\stackrel{0}{0}$} & Tierra & $3(1,3)$ & $1(0,6)$ & $4(1,0)$ \\
\hline & & Tabla & $2(0,9)$ & $1(0,6)$ & $3(0,8)$ \\
\hline & & Cemento & $125(56,6)$ & $92(54,8)$ & $217(55,8)$ \\
\hline & & Baldosa & $89(40,3)$ & $73(43,4)$ & $162(41,6)$ \\
\hline & \multirow{4}{*}{$\begin{array}{l}\stackrel{\overbrace{}}{0} \\
\stackrel{d}{\oplus}\end{array}$} & Palma & 0 & $1(0,6)$ & $1(0,3)$ \\
\hline & & Eternit & $167(75,6)$ & $128(76,8)$ & $294(75,8)$ \\
\hline & & Barro & $3(1,4)$ & $2(1,19)$ & $5(1,3)$ \\
\hline & & Zinc o lámina & $46(20,9)$ & $33(19,6)$ & $80(20,3)$ \\
\hline & \multirow{4}{*}{ 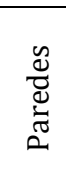 } & Bahareque & 0 & $1(0,6)$ & $1(0,3)$ \\
\hline & & Madera & $3(1,3)$ & $3(1,9)$ & $6(1,5)$ \\
\hline & & Ladrillo & $154(69,7)$ & $102(60,9)$ & $255(65,8)$ \\
\hline & & Cemento & $61(27,6)$ & $58(34,7)$ & $119(30,6)$ \\
\hline \multirow[t]{6}{*}{ Conocimientos } & \multicolumn{2}{|c|}{ Conoce la enfermedad de Chagas } & $138(62,4)$ & $98(58,4)$ & $236(60,7)$ \\
\hline & \multicolumn{2}{|c|}{ Conoce la forma de trasmisión } & $167(75,5)$ & $122(72,6)$ & $289(74,3)$ \\
\hline & \multicolumn{2}{|c|}{ Sabe si afecta alguna parte del cuerpo } & $155(70,1)$ & $111(66,1)$ & $61(68,4)$ \\
\hline & \multicolumn{2}{|c|}{ La enfermedad de Chagas tiene cura } & $52(23,5)$ & $52(30,9)$ & $103(26,7)$ \\
\hline & \multicolumn{2}{|c|}{ Sabe en qué sitios se puede encontrar el vector } & $159(71,9)$ & $122(72,6)$ & $280(72,2)$ \\
\hline & \multicolumn{2}{|c|}{ Sabe de qué se alimenta el insecto } & Si $113(51,1)$ & Si $80(47,6)$ & $192(49,6)$ \\
\hline \multirow[t]{5}{*}{ Actitudes } & \multicolumn{2}{|c|}{ Participaría en la búsqueda del vector } & $176(79,6)$ & $130(77,4)$ & $306(78,7)$ \\
\hline & \multicolumn{2}{|c|}{$\begin{array}{l}\text { Si encuentra el vector en casa, lo lleva para } \\
\text { identificación al puesto de salud }\end{array}$} & $45(20,4)$ & $33(19,6)$ & $78(20,1)$ \\
\hline & \multirow{3}{*}{\multicolumn{2}{|c|}{$\begin{array}{l}\text { Aceptaría cursos o charlas de la enfermedad } \\
\text { Si es picado por el vector iría al centro de salud } \\
\text { Aceptaría examen de sangre para saber si tiene la } \\
\text { infección }\end{array}$}} & $189(85,5)$ & $137(81,5)$ & $326(83,8)$ \\
\hline & & & $187(84,6)$ & $135(80,3)$ & $322(82,8)$ \\
\hline & & & $209(94,5)$ & $148(88,0)$ & $357(91,8)$ \\
\hline \multirow[t]{5}{*}{ Prácticas } & \multicolumn{2}{|c|}{ Ha escuchado charlas de la enfermedad } & $57(25,8)$ & $41(24,4)$ & $98(25,2)$ \\
\hline & \multicolumn{2}{|c|}{ Usa mosquitero o toldillo } & $102(46,2)$ & $83(49,4)$ & $185(47,6)$ \\
\hline & Usa & allas en puertas y ventanas & $8(4,7)$ & $10(4,5)$ & $18(4,6)$ \\
\hline & Usa & secticidas en la vivienda & $137(61,9)$ & $114(67,8)$ & $250(64,5)$ \\
\hline & Acep & aría tratamiento para la enfermedad & $217(98,2)$ & $167(99,4)$ & $383(98,7)$ \\
\hline
\end{tabular}




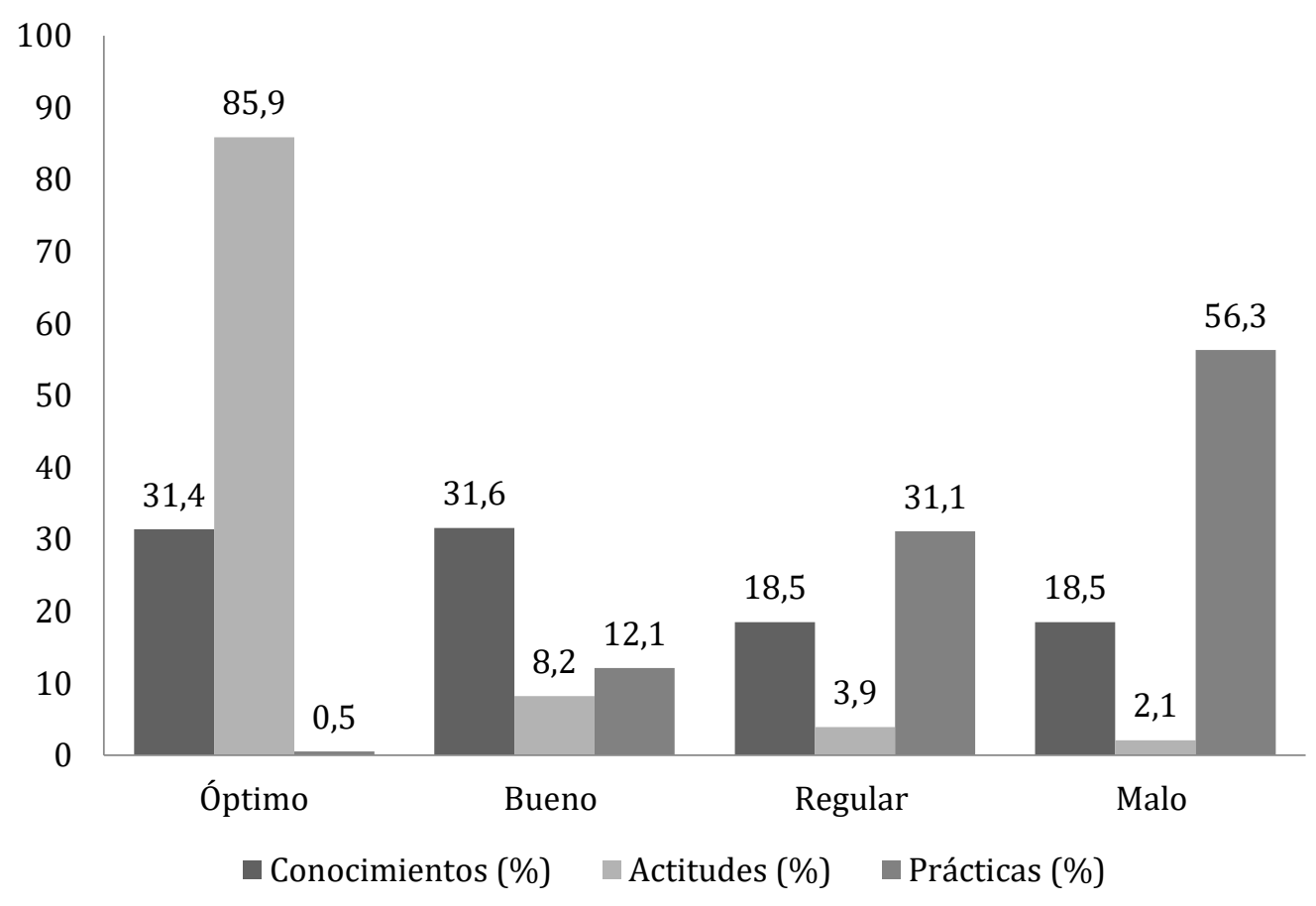

Figura 1. Resultados de CAPS en Aguazul Casanare

\section{Discusión}

La enfermedad de Chagas solía ser endémica desde el norte de México hasta Argentina(14,15), pero su presencia se ha incrementado gracias al resultado de la migración rural-urbana existiendo casos en países no endémicos(16), además de la presencia de triatomíneos silvestres y de mamíferos que se han adaptado a condiciones ambientales potenciando la trasmisión del parásito(17); situación que afecta a innumerables personas en condición vulnerabilidad(18,19). Por esto, la población está expuesta a entrar en contacto con posibles factores que deben ser evaluados y establecer su línea de base de conocimiento.

La enfermedad de Chagas a lo largo del tiempo ha tenido un fuerte impacto en la población, constituyéndose en un interés para los entes de vigilancia en salud pública, instaurando una expansión de conocimiento frente a esta patología(20), pues se debe tener en cuenta la multicausalidad de su trasmisión, entre ellas la posición de las comunidades frente a esta patología(21).

En la comunidad de Aguazul - Casanare, Colombia el $60,7 \%$ de la población tuvo conocimientos entre óptimos y buenos respecto a la enfermedad de
Chagas, que representa un porcentaje similar en relación a lo descrito en otra comunidad Casanareña en el municipio de San Luis de Palenque(22), donde se encontró que el $78,8 \%$ de los habitantes conocía la enfermedad de Chagas o había oído hablar de ella, entre tanto las prácticas fueron limitadas al igual que en el municipio de Aguazul. Además, en Aguazul el $71,5 \%$ de la comunidad reconoció el insecto vector trasmisor del parásito que es concordante con población de Pore Casanare donde el $85,7 \%$ de la población tenía algún conocimiento sobre el vector y el $72,2 \%$ ha tenido contacto con este a lo largo de su vida(23).

Adicionalmente en el presente estudio se observó que el 30,8\% de los participantes habían visto en el domicilio al vector y que el 7,9\% sabía que habían tenido contacto con este, resultados que son comparables con una investigación realizada en un área endémica de Boyacá(24) donde el $81,9 \%$ de las personas conocía el insecto vector, el $62 \%$ había visto el vector en sus casas. Sin embargo, en Boyacá cerca del 29\% de las personas afirmaron haber tenido contacto con el insecto.

En el departamento de Santander(25), se llevó a cabo un estudio sobre conocimientos de la enfermedad de Chagas en una población de estudiantes de VII y X 
semestre de enfermería, encontrando que el grado de conocimiento fue de $48,7 \%$, mayor al del presente estudio, probablemente por los diferentes niveles de educación.

Por otra parte, en la comunidad de Cárdenas, Tabasco, México, se desarrolló un estudio sobre CAPS de la enfermedad de Chagas, con una muestra total de 78 personas, al igual que en este estudio la mayor población participante fue del sexo femenino, referente a los conocimientos se observó que la población presentó conocimientos bajos $(21,8 \%)$, actitudes buenas $(85,9 \%)$ y prácticas regulares de $(48,7 \%)^{(26)}$, resultados similares a los encontrados en la comunidad Casanareña.

En Perú(27) en una comunidad escolar el 26\% de los encuestados reconoció al vector en su forma adulta, en Aguazul el porcentaje fue mayor, probablemente porque la población era mayor de edad. En Perú se identifica al parásito con el nombre de "chirimacha".

De las comunidades endémicas para esta patología se infiere que los CAPs son similares, tanto en Colombia como en otros países, sin embargo, es necesario incrementar el refuerzo educacional para optimizar las prácticas que limiten la endemia y la expansión de la patología.

Dentro de las limitaciones de la presente investigación se puede mencionar que al ser un estudio de tipo trasversal no se pueden inferir causalidad, además no se llevó a cabo cegamiento de los investigadores, también son necesarias intervenciones en la comunidad para determinar la mejora de los CAPs acerca de la enfermedad, así como su seguimiento en posteriores intervenciones.

\section{Conclusiones}

Se pudo determinar que la comunidad presenta óptimas actitudes para prevenir la infección, sin embargo, el conocimiento y sus prácticas son limitadas. Esto puede conllevar a que la población esté dispuesta a recibir información con buena actitud y mejorar sus conocimientos y prácticas de prevención frente a la infección ocasionada por el parásito que impacte a futuro en la disminución de la endemia.

Conflicto de intereses: Ninguno declarado por los autores.

\section{Referencias}

1. Arenas Monreal L, Pacheco Magaña LE, Bonilla Fernández $P$, Rueda Neria CM, Flores Alatorre JF, Caballero García CR. Procesos educativos sobre enfermedad de Chagas con escolares de nivel básico de escuelas públicas de México. Memorias del Inst Investig en Ciencias la Salud. 2018;16(1):14-8. doi: 10.18004/mem.iics/18129528/2018.016(01)14-018.

2. Ávila Montes G, Martínez Hernández M, Ponce C, Ponce E, Soto Hernández R. La enfermedad de Chagas en la zona central de Honduras: Conocimientos, creencias y prácticas. Rev Panam Salud Publica/Pan Am J Public Heal. 1998;3(3):158-63. Available from: https://www.scielosp.org/article/rpsp/1998.v3n3/158$163 /$

3. Organización Mundial de la Salud. OMS. Enfermedad de Chagas. [Internet]. 2019. [Consultado 18 Sep. 2019]. Available from: https://www.who.int/es/news-room/factsheets/detail/chagas-disease-(american-trypanosomiasis)

4. Pérez-Molina JA, Molina I. Seminar Chagas disease. The Lancet [Internet]. 2018;391(10115):82-94. Available from: http://dx.doi.org/10.1016/S0140-6736(17)31612-4

5. Chopra P, Gulwani H. Pathology and pathogenesis of rheumatic heart disease. Indian J Pathol Microbiol. 2007;50(4):685-97. Available from: https://pubmed.ncbi.nlm.nih.gov/18306530/

6. Gutierrez FRS, Trujillo Güiza ML, Escobar Martínez M del C. Prevalence of Trypanosoma cruzi Infection among People Aged 15 to 89 Years Inhabiting the Department of Casanare (Colombia). PLoS Negl Trop Dis. 2013;7(3). doi:10.1371/journal.pntd.0002113

7. Filigheddu MT, Górgolas M, Ramos JM. Orally-transmitted Chagas disease. Med Clínica (English Ed) [Internet]. 2017;148(3):125-31. Available from: http://dx.doi.org/10.1016/j.medcle.2017.02.007

8. Reyes M, Torres Á, Esteban L, Flórez M, Angulo VM. Riesgo de transmisión de la enfermedad de Chagas por intrusión de triatominos y mamíferos silvestres en Bucaramanga, Santander, Colombia. Biomedica. 2017;37(1):68-78. Available from: https://doi.org/10.7705/biomedica.v37i1.3051

9. Newton-Sánchez OA, Espinoza-Gómez F, Melnikov V, Delgado-Enciso I, Rojas-Larios F, Dumonteil E, et al. Seroprevalence of Trypanosoma cruzi (TC) and risk factors in Colima, Mexico. Gac Med Mex [Internet]. 2017;153(2):179-84. Available from: http://www.ncbi.nlm.nih.gov/pubmed/28474704

10. Paez BM, Rodriguez Riveros MI, Kasamatsu E, Castro A, Orue E, Lampert $\mathrm{N}$, et al. Conocimientos, actitudes y prácticas sobre virus de papiloma humano (VPH) y cáncer de cuello uterino en mujeres de 30 y más años de edad, de un barrio ribereño de Asunción, (Bañado Sur). 2012. Rev la Univ Ind Santander Salud. 2016;48(1):37-44. Available from: http://dx.doi.org/10.18273/revsal.v48n1-2016004

11. Orozco Ávila J, Valencia Marín A, Betancur Pérez JF. Estimation of the transfer of vascular epiphytes, as a conservation strategy in the municipality of Aguazul, Casanare, Colombia. Rev Investig Agrar y Ambient. 2017;8(1):27-37. Available from: https://doi.org/10.22490/21456453.1830

12. Montenegro D, Vera M, Zuleta L, Llanos V, Junqueira A. Estrategia para determinar la línea base en áreas de interrupción vectorial de la enfermedad de Chagas. Revista 
Panamericana de Salud Pública. 2016;39(6):341-51. Available from: https://www.scielosp.org/pdf/rpsp/2016.v39n6/341351/es

13. Suescún Carrero SH, Ramírez López LX, Sandoval Cuellar C, Monroy Díaz ÁL. Fiabilidad y validación del instrumento de conocimientos, actitudes y prácticas en la enfermedad de Chagas. Revista Cubana de Medicina Tropical. 2020;72:2. Available

from: http://revmedtropical.sld.cu/index.php/medtropical/articl e/view/461

14. Gutierrez-Daza KN, Peña Avila M, Bernal Pedraza A. Carga de la enfermedad de Chagas en el departamento de Boyacá en los años 2014 a 2016. Rev de investigación en salud. Universidad de Boyacá. 2018;5(1):68-85. Available from: https://doi.org/10.24267/23897325.288

15. Klein K, Burrone MS, Alonso JP, Rey Ares L, García Martí S, Lavenia A, et al. Estrategia para mejorar el acceso al tratamiento etiológico para la enfermedad de Chagas en el primer nivel de atención en Argentina. Rev Panam Salud Publica/Pan Am J Public Heal. 2017;41:e20. Available from: https://iris.paho.org/handle/10665.2/34029

16. Carneiro Junior N, Silveira C, da Silva LMB, Shikanai-Yasuda MA. Migração boliviana e doença de chagas: Limites na atuação do sistema único de saúde Brasileiro (SUS). Interface Commun Heal Educ. 2018;22(64):87-96. Available from: https://doi.org/10.1590/1807-57622016.0338

17. Chatelain E. Chagas disease drug discovery: Toward a new era. J Biomol Screen. 2015;20(1):22-35. Available from: https://doi.org/10.1177/1087057114550585

18. Bermudez J, Davies C, Simonazzi A, Real JP, Palma S. Current drug therapy and pharmaceutical challenges for Chagas disease. Acta Trop [Internet]. 2016;156:1-16. Available from: http://dx.doi.org/10.1016/j.actatropica.2015.12.017

19. Justi SA, Galvão C. The Evolutionary Origin of Diversity in Chagas Disease Vectors. Trends Parasitol [Internet]. 2017;33(1):42-52. Available from: http://dx.doi.org/10.1016/j.pt.2016.11.002

20. Wilches-Luna EC, Hernández NL, Hernández OM, Pérez-Vélez CM. Knowledge, attitudes, practices and education among students in a faculty of health. Rev salud pública [Internet]. 2016;18(1):129-41. Available from: http://www.scielo.org.co/scielo.php?script=sci_arttext\&pid $=$ S0124-006420160001000120
21. Ayala S, Alvarado S, Cáceres D, Zulantay I, Canals M. Estimando el efecto del cambio climático sobre el riesgo de la enfermedad de Chagas en Chile por medio del número reproductivo. Rev Med Chil. 2019;147(6):683-92. Available from: http://dx.doi.org/10.4067/S003498872019000600683

22. Castañeda-Porras O, Zuleta-Dueñas LP. Conocimientos, actitudes y prácticas para el control de enfermedades transmitidas por vectores en zona rural dispersa, San Luis de Palenque, Casanare-Colombia, 2017. Rev Médica Risaralda. 2018;24(2):108-14. Available from: http://www.scielo.org.co/scielo.php?script=sci_abstract\&pi $\mathrm{d}=\mathrm{S} 0122-06672018000200108 \& \operatorname{lng}=\mathrm{es} \& \mathrm{nrm}=\mathrm{iso}$

23. Monroy Díaz AL, Pregonero Sigua F, Otálora AS, Pedraza AM. Trypanosoma cruzi seroprevalence and associated factors in women in Casanare-Colombia. Journal of Parasitic Diseases. 2020;44:1-7. Available from: https://doi.org/10.1007/s12639-020-01280-y

24. Monroy-Díaz Á, Rodríguez-Niño S, Suescpun-Carrero SH, Ramírez-López L. Seroprevalencia de infección por Trypanosoma cruzi y factores asociados en Miraflores Boyacá, Colombia. Revista Investigación en Salud Universidad de Boyacá. 2018;5(1):31-47. Available from: https://doi.org/10.24267/23897325.284

25. Montaño-Bohórquez YA, Galvis-Pabón JS, García-Rueda A, Mensoza-Becerra JE, Roa-Díaz ZM. Evaluación del conocimiento de estudiantes de enfermería sobre la enfermedad de Chagas. Revista Científica Salud Uninorte. 2018;34(3). Available from: https://rcientificas.uninorte.edu.co/index.php/salud/articl e/view/10433

26. Ruiz-colorado MC, Rivas-acuña $V$, Gerónimo-carrillo $R$, Hernández-Ramírez G, Soancatl-Castro $M$, et al. Nivel de conocimiento y factores de riesgo de la enfermedad de Chagas en una comunidad de Cárdenas, Tabasco, México. Salud en Tabasco. 2016;22(3):61-9. Available from: https://www.redalyc.org/articulo.oa?id=48754565002

27. Cabrera R, Mayo C, Suárez N, Infante C, Náquira C, GarcíaZapata MTA. Conocimientos, actitudes y prácticas sobre la enfermedad de Chagas en población escolar de una zona endémica del Perú. Cadernos de Saúde Pública. 2003;19(1):147-54. Available from: https://doi.org/10.1590/S0102-311X2003000100016 